\title{
AN EXAMPLE OF COMPACT KÄHLER MANIFOLD WITH NONNEGATIVE QUADRATIC BISECTIONAL CURVATURE
}

\author{
QUN LI, DAMIN WU, AND FANGYANG ZHENG
}

(Communicated by Lei $\mathrm{Ni}$ )

\begin{abstract}
We construct a compact Kähler manifold of nonnegative quadratic bisectional curvature which does not admit any Kähler metric of nonnegative orthogonal bisectional curvature. The manifold is a 7-dimensional Kähler $C$ space with second Betti number equal to 1 , and its canonical metric is a Kähler-Einstein metric of positive scalar curvature.
\end{abstract}

\section{INTRODUCTION}

In recent years, the condition nonnegative quadratic bisectional curvature (which we will denote by $Q B \geq 0$ ) has drawn more and more attention; see for example [17, 9], 6], 18, and [13. At a point $p$ on a Kähler manifold $\left(M^{n}, g\right)$, this condition is defined by

$$
\sum_{i, j=1}^{n} R_{i \bar{i} j \bar{j}}\left(x_{i}-x_{j}\right)^{2} \geq 0,
$$

for any unitary tangent frame $\left\{e_{1}, \ldots, e_{n}\right\}$ at $p$ and any real numbers $x_{1}, \ldots, x_{n}$.

Note that when the bisectional curvature is nonnegative (denoted as $B \geq 0$ from now on), namely, when $R_{X \bar{X} Y \bar{Y}} \geq 0$ for any two type $(1,0)$ tangent vectors $X, Y$ at $p$, then $Q B \geq 0$ at $p$.

A condition slightly weaker than $B \geq 0$ is the so-called nonnegative orthogonal bisectional curvature, denoted as $B^{\perp} \geq 0$, which requires that $R_{X \bar{X} Y \bar{Y}} \geq 0$ for any two type $(1,0)$ tangent vectors $X, Y$ at $p$ which satisfy $X \perp Y$. Clearly, $B^{\perp} \geq 0$ already implies $Q B \geq 0$, since the diagonal terms in the summation vanish, and when $n=2, B^{\perp} \geq 0$ and $Q B \geq 0$ coincide. However, when $n \geq 3, Q B \geq 0$ does not have to have all the orthogonal bisectional curvature terms to be nonnegative; thus it is weaker than $B^{\perp} \geq 0$, at least from the algebraic point of view. It is however a totally different question whether there will be any compact Kähler manifold $\left(M^{n}, g\right)(n \geq 3)$ with $Q B \geq 0$ everywhere such that $M^{n}$ does not admit any Kähler metric with $B^{\perp} \geq 0$ everywhere.

The purpose of this note is exactly to demonstrate the existence of such a manifold.

This topic is of course closely related to the Frankel-Hartshorne conjectures, which attempt to understand the elliptic end of the high dimensional uniformization theory. The famous solution of Mok [14] to the generalized Frankel conjecture states

Received by the editors October 7, 2011.

2010 Mathematics Subject Classification. Primary 32M10, 53C55; Secondary 53C21, 53C30.

(C) 2013 American Mathematical Society 
that any compact simply connected Kähler manifold with $B \geq 0$ everywhere must be biholomorphic to a compact Hermitian symmetric space. Recently, using the Ricci flow technique and earlier work of X. X. Chen [7] and Brendle-Schoen [4, 5], $\mathrm{Gu}$-Zhang [10] proved the following result:

Theorem (Gu-Zhang). Let $\left(M^{n}, g\right)$ be a simply connected compact Kähler manifold with $B^{\perp} \geq 0$ everywhere. Then $M$ is biholomorphic to a compact Hermitian symmetric space.

In other words, the condition $B^{\perp} \geq 0$, although algebraically weaker than $B \geq 0$, does not generate any new examples, since for any compact Hermitian symmetric space, its canonical Kähler metric has $B \geq 0$ everywhere.

Here we avoided the discussion of nonsimply connected cases, since splitting theorems are already known, in the generalized Frankel case by the classic splitting theorem of Howard-Smyth-Wu 11, [16, and in the generalized Hartshorne case by Demailly-Peternell-Schneider [8].

The generalized Hartshorne conjecture seeks to understand all Fano manifolds with numerically effective tangent bundles. This class includes all the Kähler $C$ spaces, namely, all the compact simply connected homogeneous Kähler manifolds. The conjecture, in its narrowest sense, states that any compact simply connected Kählerian manifold $M^{n}$ with numerically effective tangent bundle must be biholomorphic to a Kähler $C$-space (see, for example, [19, p. 218]).

Note that for a Kähler $C$-space $M^{n}$, its canonical Kähler-Einstein metric (which is unique up to a constant multiple) has $B \geq 0$ everywhere if and only if $M^{n}$ is Hermitian symmetric. When $M^{n}$ is not Hermitian symmetric, any Kähler metric on $M^{n}$ cannot have $B \geq 0$ everywhere by Mok's Theorem. In fact, it cannot have $B^{\perp} \geq 0$ everywhere by the recent theorem of Gu and Zhang. So one has to tolerate some mild negativity of bisectional curvature in the quest of the generalized Hartshorne conjecture, at least via a differential geometric approach. In light of this, the condition $Q B \geq 0$ comes into play, and it is natural to ask whether this condition will give a good differential geometric description of Kähler $C$-spaces.

By the splitting results of $\mathrm{H}$. Wu et al., we know that under the condition $Q B \geq 0$ everywhere, any harmonic $(1,1)$ form must be parallel; thus $M^{n}$ will admit de Rham decomposition if the second Betti number $b_{2}>1$. For this reason we should restrict ourselves to Kähler $C$-spaces with $b_{2}=1$. This class includes all irreducible compact Hermitian symmetric spaces.

Let $M^{n}$ be an $n$-dimensional Kähler $C$-space with $b_{2}=1$. The homogeneous Kähler metric on $M^{n}$ is unique up to a constant multiple, and it is Kähler-Einstein. Let us denote this metric by $g_{0}$. Let $\mathcal{C}$ be the set of all Kähler $C$-spaces with $b_{2}=1$ excluding all irreducible compact Hermitian symmetric spaces.

We raise the following

Conjecture. 1) Any $\left(M^{n}, g_{0}\right)$ in $\mathcal{C}$ has $Q B \geq 0$ everywhere.

2) If $\left(M^{n}, g\right)$ is a compact simply connected and locally irreducible Kähler manifold with $Q B \geq 0$ everywhere, then $M^{n}$ is biholomorphic to a Kähler $C$-space with $b_{2}=1$.

3) In 2), $g$ is actually isometric to (a constant multiple of) $g_{0}$ if the manifold $M^{n}$ is not $\mathbb{P}^{n}$. 
In this note, we give an explicit calculation of one special example $\left(M^{7}, g_{0}\right)$ in $\mathcal{C}$ and show that it indeed has $Q B \geq 0$, thus confirming that the condition is genuinely more tolerant than $B \geq 0$ or $B^{\perp} \geq 0$. Our computation is brute-force, due to our lack of knowledge in algebra. We suspect that a more representation-theoretic computation would establish 1).

Main Theorem. The 7-dimensional Kähler $C$-space $\left(B_{3}, \alpha_{2}\right)$ has nonnegative quadratic bisectional curvature.

\section{The Curvature of KäHLER $C$-SPACES With $b_{2}=1$}

Kähler $C$-spaces were studied by H. C. Wang [15], Borel [1, 2], Borel-Hirzebruch 3], and others. A detailed study of the curvature tensor for such spaces was given by M. Itoh 12 .

Let $G$ be a simply connected, simple complex Lie group, and $\mathfrak{g}$ its Lie algebra. Fix a Cartan subalgebra $\mathfrak{h}$ of $\mathfrak{g}$. Let $l=\operatorname{dim}_{\mathbb{C}} \mathfrak{h}$, and let $\Delta$ be the root system of $\mathfrak{g}$ with respect to $\mathfrak{h}$. Fix a fundamental root system $\left\{\alpha_{1}, \ldots, \alpha_{l}\right\}$ of $\Delta$. It determines an ordering of the root system $\Delta=\Delta^{+} \cup \Delta^{-}$. We have

$$
\mathfrak{g}=\mathfrak{h} \oplus \bigoplus_{\alpha \in \Delta} \mathfrak{g}_{\alpha}
$$

where $\mathfrak{g}_{\alpha}$ is the root space corresponding to $\alpha$, satisfying $\left[\mathfrak{g}_{\alpha}, \mathfrak{g}_{\beta}\right] \subseteq \mathfrak{g}_{\alpha+\beta}$ for any two roots $\alpha, \beta \in \Delta$.

Now let us fix an integer $r$ with $1 \leq r \leq l$. Denote by

$$
\Delta_{r}^{+}(k)=\left\{\sum_{i} n_{i} \alpha_{i} \in \Delta^{+} \mid n_{r}=k\right\}, \quad \Delta_{r}^{+}=\bigcup_{k>0} \Delta_{r}^{+}(k) .
$$

Let $P \subseteq G$ be the subgroup whose Lie algebra is

$$
\mathfrak{p}=\mathfrak{h} \oplus \bigoplus_{\alpha \in \Delta \backslash \Delta_{r}^{+}} \mathfrak{g}_{\alpha} .
$$

Then $P$ is a parabolic subgroup; namely, the complex manifold $M=G / P$ is compact. This gives a Kähler $C$-space with $b_{2}=1$. Conversely, any Kähler $C$-space with $b_{2}=1$ is given this way. We will denote this space by $\left(\mathfrak{g}, \alpha_{r}\right)$.

Table 1 on page 55 of 12 gives the list of all Kähler $C$-spaces with $b_{2}=1$, using the Dynkin diagrams. The double circled ones are the Hermitian symmetric ones. From the table, we see that the simplest nonsymmetric example would be $M^{7}=\left(B_{3}, \alpha_{2}\right)$, which will be our example in this note.

In the remainder of this section, we will follow Itoh's notation and calculations 12 and collect the necessary formulas that we need later for computing the curvature of Kähler $C$-spaces with $b_{2}=1$. Let $M^{n}=\left(\mathfrak{g}, \alpha_{r}\right)$ be a Kähler $C$-space with $b_{2}=1$. Denote

$$
\mathfrak{m}_{k}^{+}=\bigoplus_{\alpha \in \Delta_{r}^{+}(k)} \mathfrak{g}_{\alpha}, \quad \mathfrak{m}^{+}=\bigoplus_{k \geq 1} \mathfrak{m}_{k}^{+},
$$

and define $\mathfrak{m}_{k}^{-}$and $\mathfrak{m}^{-}$similarly. Also, let $\mathfrak{t}=\mathfrak{h} \oplus \bigoplus_{\alpha \in \Delta_{r}^{+}(0)}\left(\mathfrak{g}_{\alpha} \oplus \mathfrak{g}_{-\alpha}\right)$. Then $\mathfrak{g}=\mathfrak{t} \oplus \mathfrak{m}$, where $\mathfrak{m}=\mathfrak{m}^{+} \oplus \mathfrak{m}^{-}$, and

$$
\left[\mathfrak{t}, \mathfrak{m}_{k}^{ \pm}\right] \subseteq \mathfrak{m}_{k}^{ \pm}, \quad\left[\mathfrak{m}_{k}^{ \pm}, \mathfrak{m}_{l}^{ \pm}\right] \subseteq \mathfrak{m}_{k+l}^{ \pm}, \quad\left[\mathfrak{m}_{k}^{+}, \mathfrak{m}_{k}^{-}\right] \subseteq \mathfrak{t}
$$

and for any $k>l>0$, it follows that $\left[\mathfrak{m}_{k}^{+}, \mathfrak{m}_{l}^{-}\right] \subseteq \mathfrak{m}_{k-l}^{+}, \quad\left[\mathfrak{m}_{k}^{-}, \mathfrak{m}_{l}^{+}\right] \subseteq \mathfrak{m}_{k-l}^{-}$. The space $\mathfrak{m}^{+}$can be identified with the holomorphic tangent space of $M^{n}$. Let $K$ be 
the Killing form of $\mathfrak{g}$. Let $E_{\alpha}$ be a Weyl canonical basis of $\mathfrak{g}$; namely, $E_{\alpha} \in \mathfrak{g}_{\alpha}$ for each $\alpha \in \Delta$, and

$$
K\left(E_{\alpha}, E_{-\alpha}\right)=-1, \quad N_{\alpha, \beta}=N_{-\alpha,-\beta},
$$

for any $\alpha, \beta \in \Delta^{+}$, where $N_{\alpha, \beta}$ is determined by $\left[E_{\alpha}, E_{\beta}\right]=N_{\alpha, \beta} E_{\alpha+\beta}$.

The canonical metric $g_{0}=\langle$,$\rangle is given by$

$$
g_{0}(X, \bar{Y})=\langle X, \bar{Y}\rangle=-k K(X, \bar{Y})
$$

for any $X, Y \in \mathfrak{m}_{k}^{+}$, where the complex conjugation on $M$ is determined by $\bar{E}_{\alpha}=$ $E_{-\alpha}$ for each $\alpha$. In the following, we will assume that

$$
X \in \mathfrak{m}_{i}^{+}, \quad Y \in \mathfrak{m}_{j}^{+}, \quad Z \in \mathfrak{m}_{k}^{+}, \quad W \in \mathfrak{m}_{l}^{+},
$$

where $i, j, k, l$ are any positive integers, and compute the curvature component $R_{X \bar{Y} Z \bar{W}}$ of the canonical metric $g_{0}$. From [12, we have

$$
R(X, \bar{Y}) Z=[\Lambda(X), \Lambda(\bar{Y})] Z-\Lambda\left([X, \bar{Y}]_{\mathfrak{m}}\right) Z-\left[[X, \bar{Y}]_{\mathfrak{t}}, Z\right],
$$

where

$$
\Lambda(X) Y=\frac{j}{i+j}[X, Y], \quad \Lambda(\bar{X}) Y=[\bar{X}, Y]_{\mathfrak{m}^{+}} .
$$

As in [12], a straightforward computation yields the following formulas.

Proposition 2.1. On a Kähler $C$-space $\left(M^{n}, g_{0}\right)$ with $b_{2}=1$, for any

$$
X \in \mathfrak{m}_{i}^{+}, \quad Y \in \mathfrak{m}_{j}^{+}, \quad Z \in \mathfrak{m}_{k}^{+}, \quad W \in \mathfrak{m}_{l}^{+},
$$

the curvature components are given by

$$
\begin{aligned}
R_{X \bar{Y} Z \bar{W}}= & (k-j) \xi_{k-j} K([X, \bar{W}],[\bar{Y}, Z])-\frac{k l}{i+k} K([X, Z],[\bar{Y}, \bar{W}]) \\
& +\left(k \xi_{i-j}+l \xi_{j-i}+l \delta_{i j} \delta_{k l}\right) K([X, \bar{Y}],[Z, \bar{W}])
\end{aligned}
$$

if $i+k=j+l$, and $R_{X \bar{Y} Z \bar{W}}=0$ otherwise. Here $\xi_{q}=1$ for $q>0$ and $\xi_{q}=0$ for $q \leq 0$.

Note that by the invariance of $K$ and the Jacobi identity, we see that the first (or the third) term on the right hand side of (2.3) can be expressed as a linear combination of the other two terms.

In the following, we will assume that $\mathfrak{g}$ satisfies the condition

$$
\Delta_{r}^{+}(k)=\emptyset, \text { for all } k \geq 3 .
$$

This condition is satisfied by all four classical sequences $A, B, C, D$ for all $r$ and for some of the exceptional cases. Note that the case $\mathfrak{m}^{+}=\mathfrak{m}_{1}^{+}$corresponds exactly to all the irreducible Hermitian symmetric cases.

For the sake of simplicity, we will assume that $M^{n}=\left(\mathfrak{g}, \alpha_{r}\right)$ is of contact type; namely, the condition (2.4) holds and $\Delta_{r}^{+}(2)$ consists of only one element. Applying Proposition 2.1 to this contact case, we get 
Proposition 2.2. Let $M^{n}$ be a Kähler $C$-space with $b_{2}=1$ of the contact type. Then for any $X, Y, Z, W$ in $\mathfrak{m}_{1}^{+}$, and $U \in \mathfrak{m}_{2}^{+}$, we have

$$
\begin{aligned}
R_{U \bar{U} U \bar{U}} & =2 K([U, \bar{U}],[U, \bar{U}]), \\
R_{X \bar{Y} U \bar{U}} & =-K([X, \bar{U}],[\bar{Y}, U])=K([X, \bar{Y}],[U, \bar{U}]), \\
R_{X \bar{Y} Z \bar{U}} & =R_{X \bar{U} U \bar{U}}=R_{X \bar{U} Z \bar{U}}=0, \\
R_{X \bar{Y} Z \bar{W}} & =-\frac{1}{2} K([X, Z],[\bar{Y}, \bar{W}])+K([X, \bar{Y}],[Z, \bar{W}]) \\
& =\frac{1}{2} K([X, Z],[\bar{Y}, \bar{W}])+K([X, \bar{W}],[Z, \bar{Y}]) .
\end{aligned}
$$

\section{The Curvature tensor of $\left(B_{3}, \alpha_{2}\right)$}

Let us now consider the specific case of $M^{7}=\left(B_{3}, \alpha_{2}\right)$, where $B_{3}=\mathfrak{s o}_{7}(\mathbb{C})$ is the Lie algebra of the Lie group $S_{7}(\mathbb{C})$. We will regard $B_{3}$ as the space of all $7 \times 7$ complex matrices $A=\left(a_{i j}\right)$ such that $a_{i j}=-a_{j^{\prime} i^{\prime}}$ for all $1 \leq i, j \leq 7$, where here and from now on we will write $i^{\prime}=8-i$. A Cartan subalgebra is given by all the diagonal matrices

$$
\mathfrak{h}=\left\{\operatorname{diag}\left(a_{1}, a_{2}, a_{3}, 0,-a_{3},-a_{2},-a_{1}\right) \mid a_{i} \in \mathbb{C}, 1 \leq i \leq 3\right\} .
$$

The root system with respect to this $\mathfrak{h}$ is

$$
\Delta=\left\{ \pm \varepsilon_{i} \pm \varepsilon_{j} \mid 1 \leq i, j \leq 3, i \neq j\right\} \cup\left\{ \pm \varepsilon_{i} \mid 1 \leq i \leq 3\right\},
$$

where $\varepsilon_{i} \in \mathfrak{h}^{*}$ is defined by

$$
\varepsilon_{i}\left(\operatorname{diag}\left\{b_{1}, b_{2}, b_{3}, 0,-b_{3},-b_{2},-b_{1}\right\}\right)=b_{i}, \quad 1 \leq i \leq 3 .
$$

Note that $\mathfrak{g}=\mathfrak{s o}_{7}(\mathbb{C})$ has three simple roots $\left\{\alpha_{1}, \alpha_{2}, \alpha_{3}\right\}$, which are given by

$$
\alpha_{1}=\varepsilon_{1}-\varepsilon_{2}, \quad \alpha_{2}=\varepsilon_{2}-\varepsilon_{3}, \quad \alpha_{3}=\varepsilon_{3} .
$$

Let $\Delta^{+}$and $\Delta^{-}$be the subsets of $\Delta$ consisting of positive and negative roots, respectively. We have

$$
\begin{aligned}
\Delta_{2}^{+}(0) & =\left\{\alpha_{1}, \alpha_{3}\right\}=\left\{\varepsilon_{1}-\varepsilon_{2}, \varepsilon_{3}\right\}, \\
\Delta_{2}^{+}(1) & =\left\{\alpha_{2}, \alpha_{2}+\alpha_{1}, \alpha_{2}+\alpha_{3}, \alpha_{2}+2 \alpha_{3}, \alpha_{2}+\alpha_{1}+\alpha_{3}, \alpha_{2}+\alpha_{1}+2 \alpha_{3}\right\} \\
& =\left\{\varepsilon_{2}-\varepsilon_{3}, \varepsilon_{1}-\varepsilon_{3}, \varepsilon_{2}, \varepsilon_{2}+\varepsilon_{3}, \varepsilon_{1}, \varepsilon_{1}+\varepsilon_{3}\right\}, \\
\Delta_{2}^{+}(2) & =\left\{2 \alpha_{2}+\alpha_{1}+2 \alpha_{3}\right\}=\left\{\varepsilon_{1}+\varepsilon_{2}\right\}, \\
\Delta_{2}^{+}(k) & =\emptyset, \quad \text { for } k \geq 3 .
\end{aligned}
$$

Therefore, $\Delta_{2}^{+}=\Delta_{2}^{+}(1) \sqcup \Delta_{2}^{+}(2)$ and $M^{7}$ is of contact type.

For convenience, let us denote by $e_{i j}$ the square matrix whose $(i, j)$-entry is 1 and other entries are zero. Let

$$
F_{i j}=e_{i j}-e_{j^{\prime} i^{\prime}}, \quad 1 \leq i, j \leq 7 .
$$

Then, $\mathfrak{h}$ is spanned by $\left\{F_{i i} \mid 1 \leq i \leq 3\right\}$ over $\mathbb{C}$. Furthermore, the root vectors can be explicitly given by

$$
\begin{aligned}
& \mathfrak{g}_{\varepsilon_{i}-\varepsilon_{j}}=\mathbb{C} F_{i j}, \quad \mathfrak{g}_{\varepsilon_{i}+\varepsilon_{j}}=\mathbb{C} F_{i j^{\prime}}, \quad \mathfrak{g}_{-\varepsilon_{i}-\varepsilon_{j}}=\mathbb{C} F_{i^{\prime} j}, \\
& \mathfrak{g}_{\varepsilon_{i}}=\mathbb{C} F_{i 4}, \quad \mathfrak{g}_{-\varepsilon_{i}}=\mathbb{C} F_{4 i}, \quad \text { for all } 1 \leq i, j \leq 3 .
\end{aligned}
$$


We compute the trace form

$$
\left(F_{i j}, F_{k l}\right)_{\mathbb{C}^{7}}=\operatorname{tr}_{\mathbb{C}^{7}}\left(F_{i j} F_{k l}\right)=2\left(\delta_{i l} \delta_{j k}-\delta_{i k^{\prime}} \delta_{j l^{\prime}}\right) .
$$

Since $\mathfrak{s o}_{7}(\mathbb{C})$ is a simple Lie algebra, any two invariant symmetric bilinear forms on $\mathfrak{s o}_{7}(\mathbb{C})$ are proportional. Thus, by rescaling some constant, we can assume that the Killing form on $\mathfrak{s o}_{7}(\mathbb{C})$ satisfies

$$
K\left(F_{i j}, F_{k l}\right)=\delta_{i l} \delta_{j k}-\delta_{j l^{\prime}} \delta_{i k^{\prime}} .
$$

Moreover, observe that

$$
\left[F_{i j}, F_{k l}\right]=\delta_{j k} F_{i l}-\delta_{i l} F_{k j}-\delta_{j^{\prime} l} F_{i k^{\prime}}-\delta_{i k^{\prime}} F_{j^{\prime} l} .
$$

We will form a unitary tangent frame $\left\{E_{1}, \ldots, E_{6} ; E_{7}\right\}$ (in the order) by

$$
\left\{F_{13}, F_{14}, F_{15}, F_{23}, F_{24}, F_{25} ; \frac{1}{\sqrt{2}} F_{16}\right\},
$$

and let their complex conjugation $\left\{\bar{E}_{1}, \ldots, \bar{E}_{6} ; \bar{E}_{7}\right\}$ be

$$
\left\{-F_{31},-F_{41},-F_{51},-F_{32},-F_{42},-F_{52} ;-\frac{1}{\sqrt{2}} F_{61}\right\} .
$$

In the following, for the benefit of calculations in the next section, we will let $a, b, c, d$ be the indices from $\{1,2\}$ and $i, j, k, l$ the indices from $\{3,4,5\}$. We will also let $X=F_{a i}, Y=F_{b j}, Z=F_{c k}, W=F_{d l}$ be vectors in $\mathfrak{m}_{1}^{+}$and $U=\frac{1}{\sqrt{2}} F_{16}$ be in $\mathfrak{m}_{2}^{+}$. Note that $F_{i j}=-F_{j^{\prime} i^{\prime}}$ for any $i, j$. By (3.2), we get

$$
\begin{aligned}
{[X, \bar{Y}] } & =\delta_{a b} F_{j i}-\delta_{i j} F_{a b}, & & {[X, Z]=-\delta_{i k^{\prime}} F_{a c^{\prime}}, } \\
{[Z, \bar{W}] } & =\delta_{c d} F_{l k}-\delta_{k l} F_{c d}, & & {[\bar{Y}, \bar{W}]=-\delta_{j l^{\prime}} F_{b^{\prime} d}, } \\
{[U, \bar{U}] } & =-\frac{1}{2}\left(F_{22}+F_{11}\right) . & &
\end{aligned}
$$

So by Proposition 2.2 and formula (3.1), we get from a straightforward computation that

$$
\begin{aligned}
R_{U \bar{U} U \bar{U}} & =2 K([U, \bar{U}],[U, \bar{U}])=1, \\
R_{X \bar{Y} U \bar{U}} & =\frac{1}{2} \delta_{i j} \delta_{a b}, \\
R_{X \bar{Y} Z \bar{W}} & =-\frac{1}{2}\left(\delta_{a d} \delta_{b c}+\delta_{a b} \delta_{c d}\right) \delta_{i k^{\prime}} \delta_{j l^{\prime}}+\delta_{a d} \delta_{b c} \delta_{i j} \delta_{k l}+\delta_{a b} \delta_{c d} \delta_{i l} \delta_{j k} .
\end{aligned}
$$

The result below follows immediately.

Proposition 3.1. The canonical metric $g_{0}$ on $M^{7}=\left(B_{3}, \alpha_{2}\right)$ is Kähler-Einstein, and its Ricci curvature is identically equal to 4.

\section{Nonnegativity of the QUadratic BiseCtional CURVATURE}

In this section, we shall show that the 7 -dimensional Kähler $C$-space $\left(M^{7}, g_{0}\right)$ given by $\left(B_{3}, \alpha_{2}\right)$ indeed has nonnegative quadratic bisectional curvature. In other words, for any unitary tangent frame $\left\{e_{a}: 1 \leq a \leq 7\right\}$ and any real numbers $x_{1}, \ldots, x_{7}$, the quantity $Q B:=\sum_{a, b=1}^{7} R\left(e_{a}, \bar{e}_{a}, e_{b}, \bar{e}_{b}\right)\left(x_{a}-x_{b}\right)^{2}$ is always nonnegative. 
Write $e_{a}=\sum_{i=1}^{7} T_{a i} E_{i}$ for some $T \in U(7)$, where $\left\{E_{1}, \ldots, E_{7}\right\}$ is the Weyl basis mentioned in $\S 3$. Since the metric $g_{0}$ is Einstein with Ricci curvature equal to 4 , we have

$$
\begin{aligned}
\frac{1}{2} Q B & =\sum_{a} R_{a \bar{a}} x_{a}^{2}-\sum_{a, b} R_{a \bar{a} b \bar{b}} x_{a} x_{b} \\
& =4 \sum_{a} x_{a}^{2}-\sum R_{i \bar{j} k \bar{l}} T_{a i} \bar{T}_{a j} T_{b k} \bar{T}_{b l} x_{a} x_{b} \\
& =4 \sum_{i, j}\left|P_{i \bar{j}}\right|^{2}-\sum_{i, j, k, l} R_{i \bar{j} k \bar{l}} P_{i \bar{j}} P_{k \bar{l}} \\
& =4|P|^{2}-\square,
\end{aligned}
$$

where $P={ }^{t} T \Lambda_{x} \bar{T}$ is a Hermitian symmetric matrix. Here we wrote $\Lambda_{x}=$ $\operatorname{diag}\left\{x_{1}, \ldots, x_{7}\right\}$ and $|P|^{2}=\sum_{i, j}\left|P_{i \bar{j}}\right|^{2}=\sum_{a} x_{a}^{2}$. Write

$$
P=\left[\begin{array}{cc}
P^{\prime} & \xi \\
t \bar{\xi} & t
\end{array}\right] .
$$

We have $|P|^{2}=\left|P^{\prime}\right|^{2}+2|\xi|^{2}+t^{2}$. Since under the Weyl frame $\left\{E_{i}\right\}$, we have $R_{7 \overline{7} 7 \overline{7}}=1$ and $R_{i \bar{j} 7 \overline{7}}=\frac{1}{2} \delta_{i j}$ for any $1 \leq i, j \leq 6$, we know that

$$
\begin{aligned}
\square & =\sum_{i, j, k, l=1}^{6} R_{i \bar{j} k \bar{l}} P_{i \bar{j}} P_{k \bar{l}}+t^{2}+t \cdot \operatorname{tr}\left(P^{\prime}\right)+|\xi|^{2} \\
& =\square^{\prime}+t^{2}+t \cdot \operatorname{tr}\left(P^{\prime}\right)+|\xi|^{2} .
\end{aligned}
$$

Plugging these into the formula for $Q B$, we get

$$
\frac{1}{2} Q B=7|\xi|^{2}+3 t^{2}-t \cdot \operatorname{tr}\left(P^{\prime}\right)+\left(4\left|P^{\prime}\right|^{2}-\square^{\prime}\right) .
$$

So $Q B \geq 0$ for any Hermitian matrix $P$ is equivalent to

$$
\Phi:=4\left|P^{\prime}\right|^{2}-\square^{\prime}-\frac{1}{12}\left(\operatorname{tr}\left(P^{\prime}\right)\right)^{2} \geq 0
$$

for any $6 \times 6$ Hermitian matrix $P^{\prime}$.

Writing $\left\{E_{1}, \ldots, E_{6}\right\}$ as $\left\{F_{a i}: 1 \leq a \leq 2,3 \leq i \leq 5\right\}$ and using formula (3.3), we get

$$
\begin{aligned}
\square^{\prime}=\sum_{a, b=1}^{2} \sum_{i, k=3}^{5}\left(-\frac{1}{2} P_{a i \overline{a k}} P_{b i^{\prime} \overline{b k^{\prime}}}\right. & -\frac{1}{2} P_{a i \overline{b k}} P_{b i^{\prime} \overline{a k^{\prime}}} \\
& \left.+P_{a i \overline{b i}} P_{b k \overline{a k}}+P_{a i \overline{a k}} P_{b k \overline{b i}}\right) .
\end{aligned}
$$

Here as before, $i^{\prime}=8-i$, and we use double indices for $P$, e.g., $P_{13 \overline{13}}=P_{1 \overline{1}}$. Now let $A, B, C$ be the $3 \times 3$ matrices given by

$$
A_{i j}=P_{1 i \overline{1 j}}, \quad B_{i j}=P_{1 i \overline{2 j}}, \quad C_{i j}=P_{2 i \overline{2 j}} ;
$$

then we can write $\square^{\prime}$ as

$$
\begin{aligned}
& -\frac{1}{2}\langle A+C, A+C\rangle-\frac{1}{2}\left(\langle A, A\rangle+\langle C, C\rangle+\left\langle B, B^{*}\right\rangle+\left\langle B^{*}, B\right\rangle\right) \\
& +(\operatorname{tr} A)^{2}+(\operatorname{tr} C)^{2}+2|\operatorname{tr} B|^{2}+|A+C|^{2},
\end{aligned}
$$


where $|A|^{2}=\sum_{i, j=3}^{5}\left|A_{i j}\right|^{2},\langle A, B\rangle=\sum_{i, j=3}^{5} A_{i j} B_{i^{\prime} j^{\prime}}=\langle B, A\rangle$, and $B^{*}={ }^{t} \bar{B}$. Since $\operatorname{tr}\left(P^{\prime}\right)=\operatorname{tr} A+\operatorname{tr} C$, and $\left|P^{\prime}\right|^{2}=|A|^{2}+|C|^{2}+2|B|^{2}$, we get

$$
\begin{aligned}
\Phi & =4\left(|A|^{2}+|C|^{2}+2|B|^{2}\right)-\square^{\prime}-\frac{1}{12}(\operatorname{tr} A+\operatorname{tr} C)^{2} \\
& =\Phi_{1}+\Phi_{2},
\end{aligned}
$$

where

$$
\begin{aligned}
\Phi_{1}= & 4|A|^{2}+4|C|^{2}+\langle A, A\rangle+\langle C, C\rangle+\langle A, C\rangle-(\operatorname{tr} A)^{2} \\
& -(\operatorname{tr} C)^{2}-|A+C|^{2}-\frac{1}{12}(\operatorname{tr} A+\operatorname{tr} C)^{2}, \\
\Phi_{2}= & 8|B|^{2}+\left\langle B, B^{*}\right\rangle-2|\operatorname{tr} B|^{2} .
\end{aligned}
$$

From

$$
\left\langle B, B^{*}\right\rangle=\left|B_{44}\right|^{2}+\left|B_{35}\right|^{2}+\left|B_{53}\right|^{2}+2 \operatorname{Re}\left(B_{33} \bar{B}_{55}+B_{34} \bar{B}_{45}+B_{43} \bar{B}_{54}\right),
$$

we see that $\left\langle B, B^{*}\right\rangle \geq-|B|^{2}$. This together with the fact that $|\operatorname{tr} B|^{2} \leq 3|B|^{2}$ implies that

$$
\Phi_{2} \geq(8-1-6)|B|^{2} \geq 0 \text {. }
$$

To deal with $\Phi_{1}$, let us write $\Phi_{1}=\Phi_{1}^{\prime}+\Phi_{1}^{\prime \prime}$, where $\Phi_{1}^{\prime}$ is the part of $\Phi_{1}$ coming from the (34), (43), (45), and (54) entries of $A$ and $C$, and $\Phi_{1}^{\prime \prime}$ is the rest. Then, we have

$$
\begin{aligned}
\Phi_{1}^{\prime}= & 8\left|A_{34}\right|^{2}+8\left|A_{45}\right|^{2}+8\left|C_{34}\right|^{2}+8\left|C_{45}\right|^{2}+4 \operatorname{Re}\left(A_{34} \bar{A}_{45}+C_{34} \bar{C}_{45}\right) \\
& +2 \operatorname{Re}\left(A_{34} \bar{C}_{45}+C_{34} \bar{A}_{45}\right)-2\left|A_{34}+C_{34}\right|^{2}-2\left|A_{45}+C_{45}\right|^{2} .
\end{aligned}
$$

Using the Cauchy-Schwarz inequality, it is easy to see that

$$
\Phi_{1}^{\prime} \geq\left(\left|A_{34}\right|^{2}+\left|A_{45}\right|^{2}+\left|C_{34}\right|^{2}+\left|C_{45}\right|^{2}\right) \geq 0 .
$$

So we are only left with the verification of the nonnegativity of $\Phi_{1}^{\prime \prime}$. Let us denote by $a=\left(a_{1}, a_{2}, a_{3}\right)$ the three diagonal elements of $A$, let $c=\left(c_{1}, c_{2}, c_{3}\right)$ be the three diagonal elements of $C$, and write $x=A_{35}, y=C_{35}$. Then we have $\Phi_{1}^{\prime \prime}=\Phi_{11}+\Phi_{12}$, where

$$
\begin{aligned}
\Phi_{11} & =10|x|^{2}+10|y|^{2}+2 \operatorname{Re}(x \bar{y})-2|x+y|^{2}, \\
\Phi_{12} & =4|a|^{2}+4|c|^{2}+\left(2 a_{1} a_{3}+a_{2}^{2}\right)+\left(2 c_{1} c_{3}+c_{2}^{2}\right)+\left(a_{1} c_{3}+a_{3} c_{1}+a_{2} c_{2}\right) \\
& -t_{a}^{2}-t_{c}^{2}-|a+c|^{2}-\frac{1}{12}\left(t_{a}+t_{c}\right)^{2},
\end{aligned}
$$

where $t_{a}=a_{1}+a_{2}+a_{3}$ and $|a|^{2}=a_{1}^{2}+a_{2}^{2}+a_{3}^{2}$, and likewise for $c$. Clearly, $\Phi_{11} \geq 0$, and $\Phi_{12}$ is a homogeneous polynomial of degree 2 in $a_{i}$ and $c_{i}, i=1,2,3$. It is not hard to see that $\Phi_{12}=(a, c) D^{t}(a, c)$, where $D$ is the real $6 \times 6$ symmetric matrix given by

$$
D=\left[\begin{array}{cc}
G & H \\
H & G
\end{array}\right]
$$

in which $G=3 I-\frac{13}{12} L+J, H=-I-\frac{1}{12} L+\frac{1}{2} J$, with

$$
L=\left[\begin{array}{lll}
1 & 1 & 1 \\
1 & 1 & 1 \\
1 & 1 & 1
\end{array}\right], \quad J=\left[\begin{array}{lll}
0 & 0 & 1 \\
0 & 1 & 0 \\
1 & 0 & 0
\end{array}\right],
$$


and $I=I_{3}$ the identity matrix. We want to show that $D \geq 0$, or equivalently, that the $3 \times 3$ matrix

$$
G-H G^{-1} H \geq 0 .
$$

Consider the matrix $T \in O(3)$ given by

$$
T=\frac{1}{\sqrt{6}}\left[\begin{array}{ccc}
\sqrt{3} & 1 & \sqrt{2} \\
0 & -2 & \sqrt{2} \\
-\sqrt{3} & 1 & \sqrt{2}
\end{array}\right]
$$

We have

$$
{ }^{t} T L T=3\left[\begin{array}{lll}
0 & 0 & 0 \\
0 & 0 & 0 \\
0 & 0 & 1
\end{array}\right], \quad{ }^{t} T J T=\left[\begin{array}{ccc}
-1 & 0 & 0 \\
0 & 1 & 0 \\
0 & 0 & 1
\end{array}\right] .
$$

Therefore, we get

$$
{ }^{t} T G T=\left[\begin{array}{ccc}
2 & 0 & 0 \\
0 & 4 & 0 \\
0 & 0 & \frac{3}{4}
\end{array}\right], \quad{ }^{t} T H T=\left[\begin{array}{ccc}
-\frac{3}{2} & 0 & 0 \\
0 & -\frac{1}{2} & 0 \\
0 & 0 & -\frac{3}{4}
\end{array}\right] .
$$

Thus,

$$
{ }^{t} T\left(G-H G^{-1} H\right) T=\left[\begin{array}{ccc}
2 & 0 & 0 \\
0 & 4 & 0 \\
0 & 0 & \frac{3}{4}
\end{array}\right]-\left[\begin{array}{ccc}
\frac{9}{8} & 0 & 0 \\
0 & \frac{1}{16} & 0 \\
0 & 0 & \frac{3}{4}
\end{array}\right] \geq 0 .
$$

This establishes the nonnegativity of $\Phi_{12}$, hence the entire $\Phi$, and by (4.1) we have shown that $\left(B_{3}, \alpha_{2}\right)$ has nonnegative quadratic bisectional curvature.

\section{REFERENCES}

[1] A. Borel, Kählerian coset spaces of semi-simple Lie groups, Proc. Nat. Acad. Sci. U.S.A., 40 (1954), 1147-1151. MR0077878 (17:1108e)

[2] A. Borel, On the curvature tensor of Hermitian symmetric manifolds, Ann. of Math. (2), 71 (1960), 508-521. MR0111059 (22:1923)

[3] A. Borel and F. Hirzebruch, Characteristic classes and homogeneous spaces I, Amer. J. Math., 80 (1958), 458-538. MR0102800 (21:1586)

[4] S. Brendle and R. Schoen, Classification of manifolds with weakly 1/4-pinched curvature, Acta. Math., 200 (2008), 1-13. MR2386107 (2009h:53087)

[5] S. Brendle and R. Schoen, Manifolds with 1/4-pinched curvature are space forms, J. Amer. Math. Soc., 22 (2009), 287-307. MR2449060(2010a:53045)

[6] A. Chau and L. F. Tam, On quadratic orthogonal bisectional curvature, preprint. To appear in J. Differential Geom.

[7] X. X. Chen, On Kähler manifolds with positive orthogonal bisectional curvature, Adv. Math., 215 (2007), 427-445. MR 2355611(2008h:32029)

[8] J. P. Demailly, T. Peternell, and M. Schneider, Compact complex manifolds with numerically effective tangent bundles, J. Algebraic Geom., 3 (1994), 295-345. MR1257325 (95f:32037)

[9] J. X. Fu, Z. Wang, and D. Wu, Form-type equations on Kähler manifolds of nonnegative orthogonal bisectional curvature, arXiv: 1010.2022

[10] H. L. Gu and Z. H. Zhang, An extension of Mok's Theorem on the generalized Frankel conjecture, Sci. China, Math., 53 (2010), 1253-1264. MR.2653275 (2011d:53156)

[11] A. Howard, B. Smyth, and H. Wu, On compact Kähler manifolds of nonnegative bisectional curvature, I, Acta. Math., 147 (1981), 51-56. MR631087 (83e:53064a)

[12] M. Itoh, On curvature properties of Kähler C-spaces, J. Math. Soc. Japan, 30 (1978), 39-71. MR.0470904 (57:10648)

[13] Q. Li, D. Wu, and F. Zheng, Quadratic bisectional curvature and constant rank theorems, in preparation. 
[14] N. Mok, The uniformization theorem for compact Kähler manifolds of nonnegative holomorphic bisectional curvature, J. Differential Geom., 27 (1988), 179-227. MR925119|(89d:53115)

[15] H. C. Wang, Closed manifolds with homogeneous complex structures, Amer. J. Math., 76 (1954), 1-32. MR.0066011 (16:518a)

[16] H. Wu, On compact Kähler manifolds of nonnegative bisectional curvature, II, Acta. Math., 147 (1981), 57-70. MR631088 (83e:53064b)

[17] D. Wu, S. T. Yau and F. Zheng, A degenerate Monge-Ampère equation and the boundary classes of Kähler cones, Math. Res. Lett., 16 (2009), 365-374. MR2496750 (2010c:32061)

[18] X. Zhang, On the boundary of Kähler cones, Proc. Amer. Math. Soc., 140 (2012), 701-705. MR2846339

[19] F. Zheng, Complex Differential Geometry, AMS and International Press, 2000. MR.1777835 (2001i:32035)

Department of Mathematics and Statistics, Wright State University, 3640 Colonel Glenn Highway, Dayton, Ohio 45435

E-mail address: qun.li@wright.edu

Department of Mathematics, The Ohio State University, 1179 University Drive, NEWARK, OHIO 43055

E-mail address: dwu@math.ohio-state.edu

Current address: Department of Mathematics, University of Connecticut, Storrs, Connecticut 06269

E-mail address: damin.wu@uconn.edu

Department of Mathematics, The Ohio State University, 231 West 18th Avenue, Columbus, Ohio 43210 - And - Center for Mathematical Sciences, Zhejiang University, Hangzhou, 310027 People's Republic of China

E-mail address: zheng@math.ohio-state.edu 